\begin{tabular}{|c|c|}
\hline Title & Introduction of the harmonic distortion determining factor and its application to evaluating real time PWM inverters \\
\hline Author(s) & Fukuda, S.; Iwaji, K. \\
\hline Citation & $\begin{array}{l}\text { IEEE Transactions on Industry A pplications, 31(1), 149-154 } \\
\text { https://doi.org/10.1109/28.363037 }\end{array}$ \\
\hline Issue Date & 1995 \\
\hline Doc URL & http:/hdl .handle.net/2115/6076 \\
\hline Rights & $\begin{array}{l}\text { (1) } 1995 \text { IEEE. Personal use of this material is permitted. However, permission to reprint/republish this material for } \\
\text { advertising or promotional purposes or for creating new collective works for resale or redistribution to servers or lists, } \\
\text { or to reuse any copyrighted component of this work in other works must be obtained from the IEEE." } \\
\text { IEEE, IEEE Transactions on Industry A pplications, } \\
\text { 1995, V olume: } 31 \text {, Issue: } 1 \text {, page(s): } 149-154\end{array}$ \\
\hline Type & article \\
\hline File Information & ITIA 31-1.pdf \\
\hline
\end{tabular}

Instructions for use 


\title{
Introduction of the Harmonic Distortion Determining Factor and Its Application to Evaluating Real Time PWM Inverters
}

\author{
Shoji Fukuda, Member, IEEE, and Yoshitaka Iwaji
}

\begin{abstract}
Frequency spectra of inverter output currents are one of the important factors in order to evaluate PWM methods. These spectra are, however, influenced not only by the PWM method itself but by the operating conditions of the inverter such as the switching frequency or load parameters. The harmonic distortion determining factor (HDDF) is considered to be a common quality index that represents the intrinsic spectral property of individual PWM methods. As it has a close relation to RMS values of the harmonic current or torque ripples of driven motors and, further, it is almost independent of the operating conditions, HDDF is quite useful for evaluating PWM methods. In this paper three typical analog PWM methods and four digital PWM methods are compared and evaluated based on HDDF values.
\end{abstract}

\section{INTRODUCTION}

M ANY real time PWM methods for inverter control have been proposed [1]-[4]. The spectral property of the inverter output current is one of the important factors used to evaluate a PWM method. Evaluation of PWM methods is, however, not easy because the frequency spectra of the inverter current are greatly influenced by the switching frequency and load parameters of the inverter. For instance, if the switching frequency is very high or the load inductance is very large compared to the load resistance, the current spectra will be excellent irrespective of the PWM method.

Therefore, a common quality index which is independent of the inverter operating conditions such as the switching frequency and load parameters is desirable for evaluating a selected PWM method. The harmonic distortion determining factor (HDDF), a function of the modulation index of the inverter, is one candidate for such a quality index.

HDDF is also useful for predicting the harmonic properties of PWM inverters. For instance, the harmonic characteristics such as the spectra of motor current harmonics or torque ripples in the case of ac drives are usually obtained by Fourier transformation or simulation. However, if HDDF values for individual PWM's are known, the approximate

Paper IPCSD 94-59, approved by the Industrial Power Converter Committee of the IEEE Industrial Applications Society for presentation at the 1993 Industrial Applications Society Annual Meeting, Toronto, Ontario, Canada, October 3-8, and the IEEJ Technical Meeting on Semiconductor Power Converter, Naha, Okinawa, Japan, Oct. 25-26, 1994. Manuscript released for publication July 21, 1994.

S. Fukuda is with the Department of Electrical Engineering, Hokkaido University, Sapporo, 060 Japan.

Y. Iwaji is with Hitachi Research Laboratory, Hitachi Ltd., Ibaraki, 319-12 Japan.

IEEE Log Number 9406604. harmonic characteristics are easily calculated by using the HDDF values.

In this paper, the basic idea and physical meaning of HDDF is first described by means of a simple example, a dc/dc down converter. Then the idea of HDDF is expanded to include three-phase PWM inverters. Next, the relation of HDDF to the RMS value of 'motor current harmonics or torque ripples in the case of ac drives, and the relation of HDDF to the RMS value of ac side harmonic current or dc side harmonic voltage in the case of rectifier operation are described. In short, HDDF is a normalized representation of the RMS distortion current, which has been used in [5] for comparing two PWM's in the case of an IM drive, the sine PWM and the space vector PWM. Here, however, two kind HDDF's, $d$-axis HDDF and total HDDF, are introduced and used. Finally HDDF curves under variable output voltage conditions are illustrated for typical real time PWM's, three analog PWM's and four digital PWM's, and evaluations of these PWM methods based on the HDDF values are given.

\section{BASIC IDEA OF HDDF}

To clarify the basic idea of HDDF, consider the dc/dc down converter shown in Fig. 1. We know empirically the following facts concerning RMS value of the ac or harmonic component of the load current, $I_{h}$ :

1) $I_{h}$ is directly proportional to the dc supply voltage $V_{\mathrm{dc}}$.

2) $I_{h}$ is almost inversely proportional to the inductance $L$.

3) $I_{h}$ is almost inversely proportional to the switching or chopping frequency $\omega_{s}$.

4) $I_{h}$ is almost independent of the resistance $R$.

5) $I_{h}$ is almost independent of the dc load current $I_{\mathrm{dc}}$.

From these facts, the ac current will be approximately represented by

$$
I_{h}=\frac{\searrow_{\mathrm{dc}}}{\omega_{s} L} P(M)
$$

where $P$, which is only a function of the duty ratio, $M=$ $t_{\mathrm{on}} / T$, is called the harmonic distortion determining factor of the $\mathrm{dc} / \mathrm{dc}$ converter. If $\omega_{s} L \gg R,(1)$ gives us a good approximation of the RMS value of the load harmonic current.

Let us derive (1). The switching function, $S$, of the $\mathrm{dc} / \mathrm{dc}$ converter in Fig. 1 is defined as:

$S=1$ when the transistor is $\mathrm{ON}$,

$S=0$ when the transistor is OFF. 


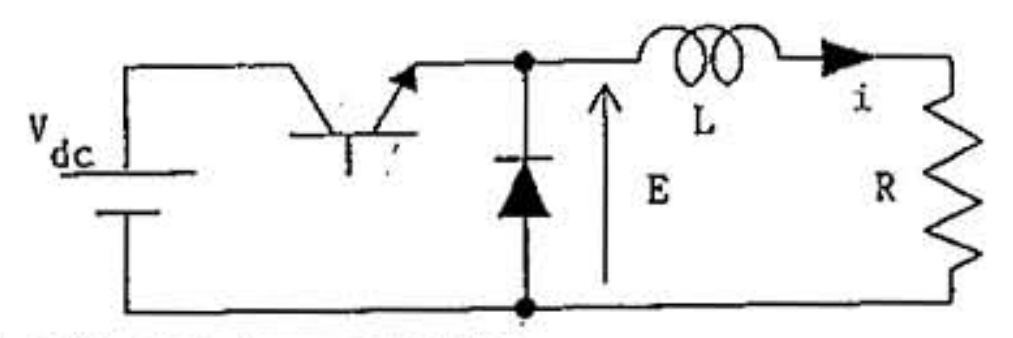

Fig. 1. A dc/dc step down converter.

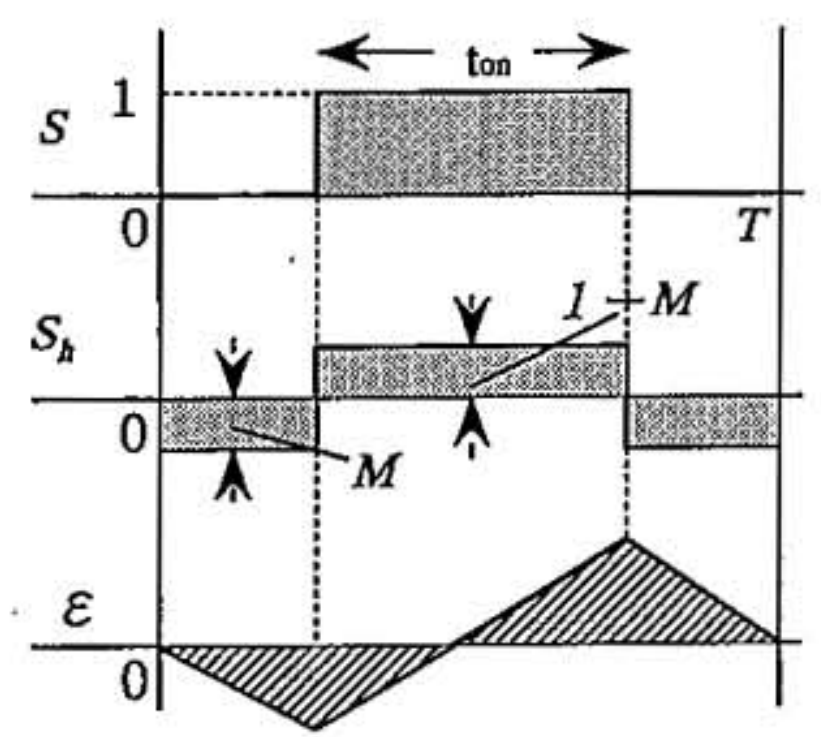

Fig. 2. Normalized voltage pulses and resulting harmonic current within one chopping period.

If the chopping frequency, $\omega_{s}$, is high enough, then $\omega_{s} L \gg$ $R$, and the ac load current $i_{h}$ will be determined by:

$$
L \cdot \frac{d}{d t} i_{h}=E-M V_{\mathrm{dc}}=V_{\mathrm{dc}}(S-M)=V_{\mathrm{dc}} S_{h}
$$

where $S_{h}$ denotes the ac or harmonic component of SF. Fig. 2 shows the waveforms of $S$ and $S_{h}$ for one chopping interval, $T$. Solving (2) we have

$$
i_{h}=\frac{V_{\mathrm{dc}}}{L} \varepsilon, \varepsilon=\int S_{h} d t
$$

where $\epsilon$ is the normalized ac component of the load current, and its waveform is shown in Fig. 2.

Now define HDDF of the dc/dc converter as:

$$
P=\omega_{s} \sqrt{\frac{1}{T} \int_{0}^{T} \varepsilon^{2} d t}=\frac{\pi(1-M) M}{\sqrt{3}}
$$

where $T$ denotes the chopping interval. Then the RMS of $i_{h}$ is given by (1). The ac current $I_{h}$ for any $M$ values is easily calculated if $P$ and the operating conditions, $\omega_{s}, L$ and $V_{\mathrm{dc}}$, are given. It is noted that $P$ is independent of the operating parameters of the $\mathrm{dc} / \mathrm{dc}$ converter.

\section{HDDF FOR THREE PHASE PWM INVERTERS}

Here the idea of HDDF is expanded to include a three-phase inverter. Consider the PWM voltage source inverter shown in Fig. 3 which is supplying ac power to an inductive load.

\section{A. Switching Function}

The switching function (SF) of the inverter for phase "a" is defined as:

$S_{\mathrm{a}}=1$ when the upper arm switch $S_{\mathrm{a}+}$ is ON,

$S_{\mathrm{a}}=-1$ when the lower arm switch $S_{\mathrm{a}^{-}}$is ON.

SF's for phase " $b$ " and " $c$ " are also defined similarly. The output potentials of the inverter are obtained by multiplying SF by the dc source voltage $V_{\text {dc }}$.

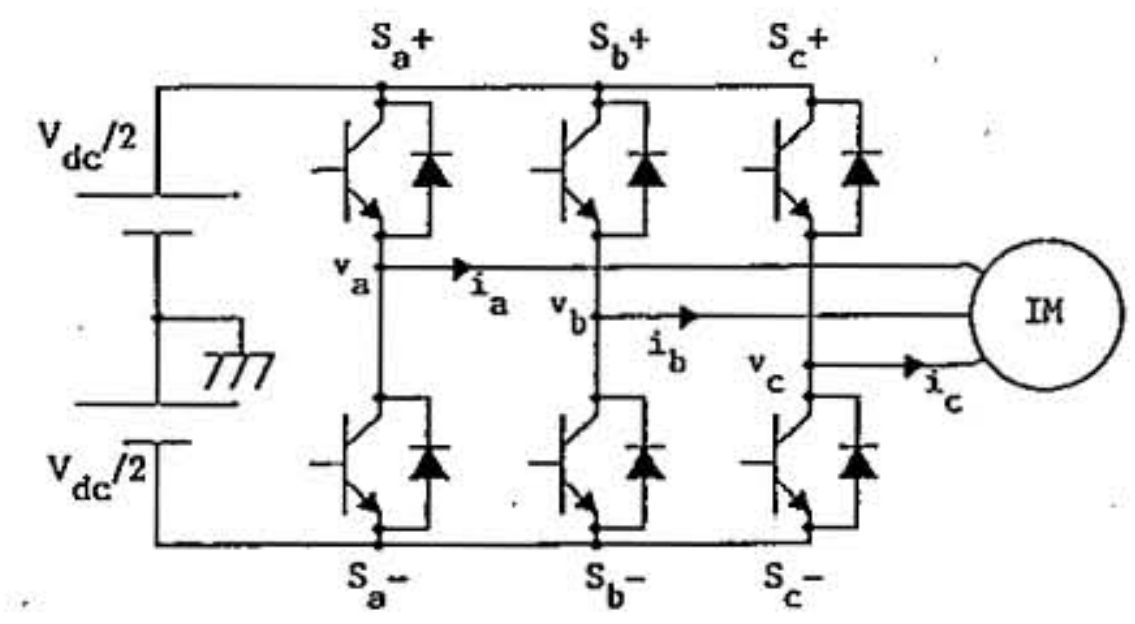

Fig. 3. A voltage source inverter.

\section{B. Transformation of the $S F$ into the $d-q$ Coordinate}

$S_{\mathrm{a}}, S_{\mathrm{b}}, S_{\mathrm{c}}$ in the real axis are transformed into $S_{d}$ and $S_{q}$ in the $d-q$ axis, which is rotating at an angular inverter output frequency, $\omega$. The fundamental component of the inverter output will then be represented by a dc component. Thus, situations similar to those of the $\mathrm{dc} / \mathrm{dc}$ converter are obtained. The $q$ axis is selected to make its dc component equal to zero. Let $S_{d h}$ and $S_{q h}$ be the ac components of $S_{d}$ and $S_{q}$, respectively. $S_{d h}$ and $S_{q h}$ correspond to the harmonic voltages of the inverter output.

\section{Definition of HDDF}

If the switching frequency, $\omega_{s}$, is high enough, then $\omega_{s} L \gg$ $R$, and $d$-axis harmonic current, $i_{d h}$, will be given by:

$$
i_{d h}=\frac{V_{\mathrm{dc}}}{L} \varepsilon_{d}, \quad \varepsilon_{d}=\int S_{d h} d t .
$$

Then, HDDF's in the $d$ and $q$ axes are defined by expressions similar to (4):

$$
\left.\begin{array}{l}
P_{d}=\frac{\omega_{s}}{\sqrt{2}} \sqrt{\frac{1}{T} \int_{0}^{T} \varepsilon_{d}^{2} d t}, \quad P_{q}=\frac{\omega_{s}}{\sqrt{2}} \sqrt{\frac{1}{T} \int_{0}^{T} \varepsilon_{q}^{2} d t} \\
P=\sqrt{P_{d}^{2}+P_{q}^{2}}
\end{array}\right\}
$$

where $T$ denotes one-sixth of an inverter output period. As the switching function is determined in relation to the modulation index, $M, P_{d}$ and $P_{q}$ are primarily functions of $M$. It has been confirmed that neither $P_{d}$ nor $P_{q}$ depends on the frequency ratio, $\omega_{s} / \omega$, if $\omega_{s} / \omega$ is larger than 40 .

\section{RELATION BETWEEN HDDF AND INVERTER PERFORMANCE}

\section{A. Induction Motor Drives}

The prediction of harmonic characteristics of ac drives such as the RMS of motor current or torque pulsations is easily done by using HDDF. In the case of induction motor drives, we have shown [6], [7] that the RMS values of the input harmonic current, $I_{h}$, and torque ripples, $T_{h}$ of the driven motor are approximately obtained by

$I_{h}=\sqrt{\sum_{k=2}^{\infty} I_{k}^{2}}=\frac{V_{\mathrm{dc}}}{2 \omega_{s} l} P, \quad T_{h}=\sqrt{\sum_{k=1}^{\infty} T_{k}^{2}}=\frac{M V_{\mathrm{dc}}^{2}}{2 \sqrt{2} \omega_{s} \omega l} P_{d}$

where $l$ denotes the leakage inductance of the motor per phase, and $I_{k}$ and $T_{k}$ denote the RMS values of the $k$-th harmonic current and the $k$-th harmonic torque, respectively. $I_{h}$ and $T_{h}$ values obtained by simulation and by HDDF are compared 


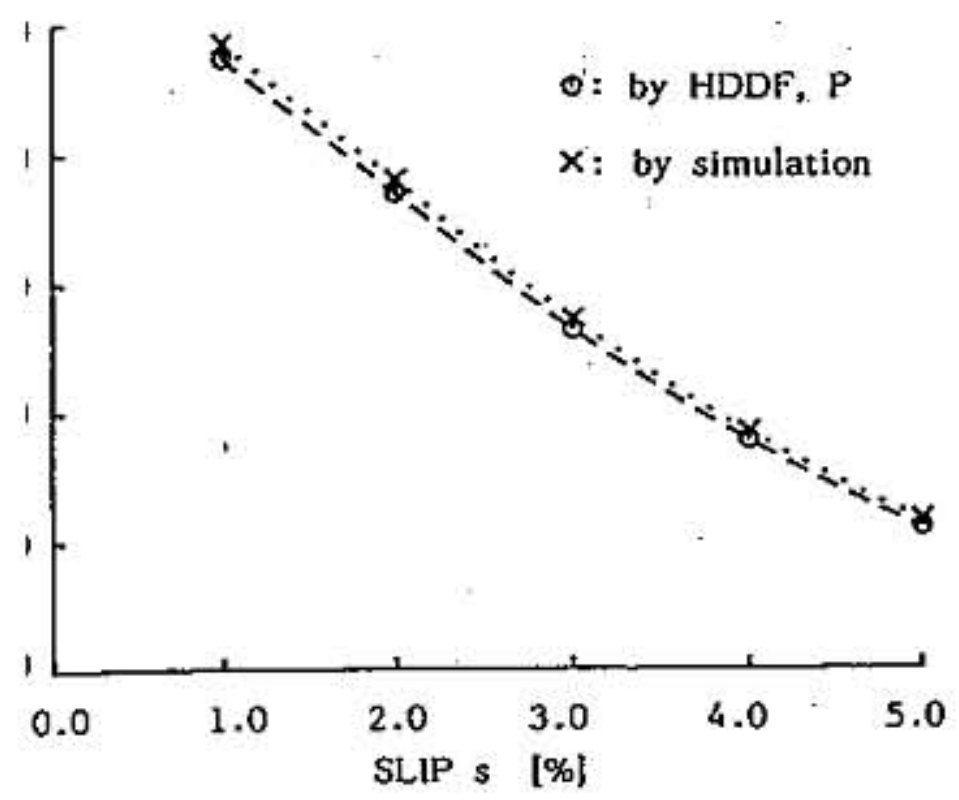

(a)

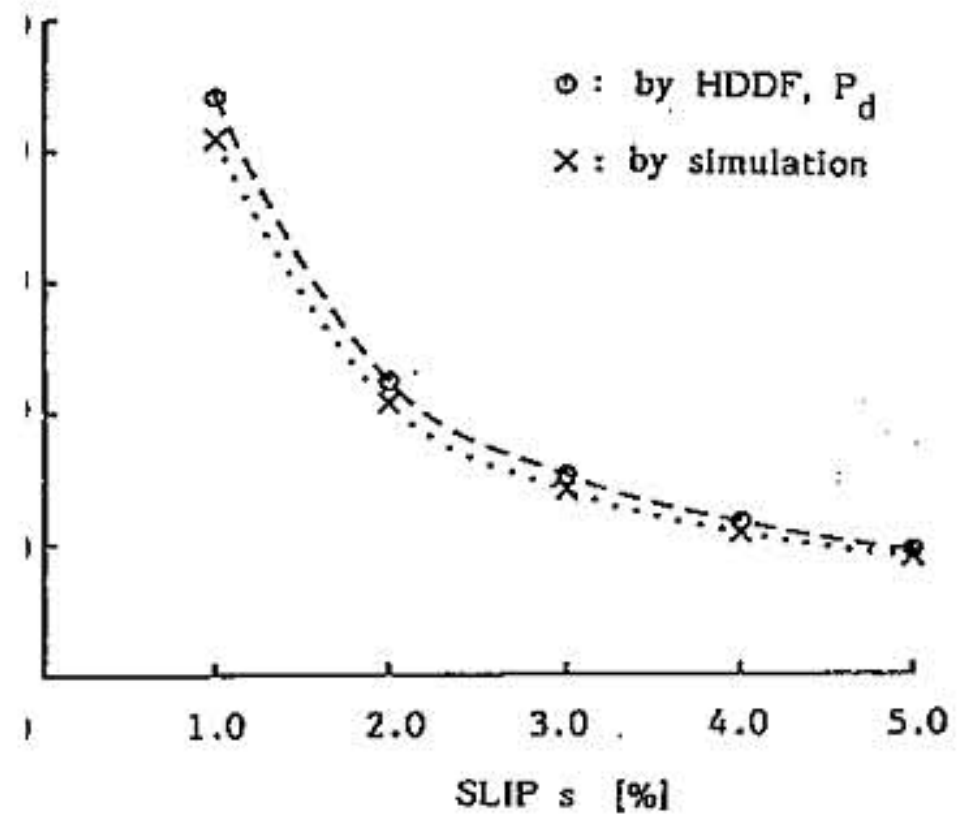

(b)

son of RMS values in ac drives obtained by simulation and otor current harmonics. (b) Torque ripples.

ere the original sine-triangular PWM is used; ratio is $\omega_{s} / \omega=36$ and the modulation index stant. Definitions of. THD are:

$$
\mathrm{THD}_{i}=\frac{I_{h}}{I_{1}}, \quad \mathrm{THD}_{t}=\frac{T_{h}}{T_{0}}
$$

$T_{0}$ denote the RMS values of the motor funda$i$ and the motor average torque, respectively. The an example has the following ratings: $0.75[\mathrm{~kW}]$, $50[\mathrm{~Hz}], f_{s}=1.8[\mathrm{kHz}]$ and $V_{\mathrm{dc}}=141[\mathrm{~V}]$ r constants; $L_{1}=L_{2}=15.1[\mathrm{mH}], M_{m}=146$ L $[\Omega]$, and $R_{2}=1.3[\Omega]$. It is seen that both the ad by simulation and by HDDF are very close

\section{ige Source Rectifiers}

feature of a PWM voltage source rectifier is it dc voltage can be controlled while keeping ic input current. In this situation, the induction : source, $V_{\mathrm{dc}}$ in Fig. 3 are replaced by an ac $\mathrm{d}$ the parallel combination of a filter capacitor, luctive load, $R_{1}+L_{1}$, respectively. Power flow Im the ac side to the dc side. We have shown [8], of the input harmonic current, $I_{h}$, and RMS of monic voltage, $V_{h}$, are approximately given by

$I_{h}=\frac{V_{\mathrm{dc} 0}}{\omega_{s} L} P, \quad V_{h}=\frac{I_{\mathrm{d} 0}}{\omega_{s} C} P_{d}$

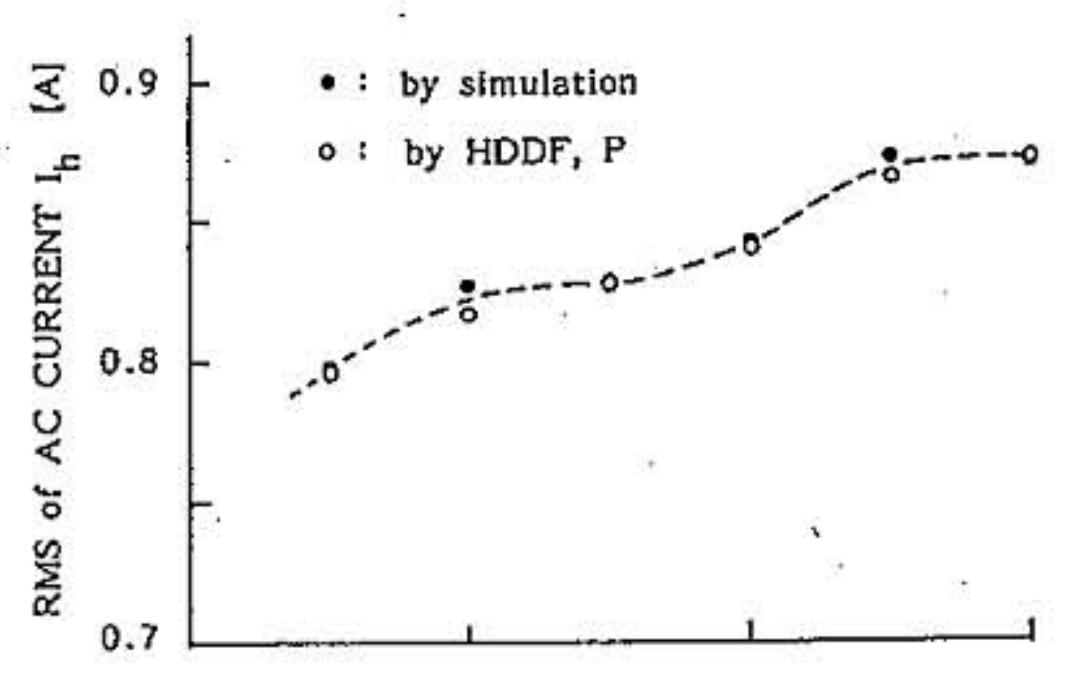

(a)

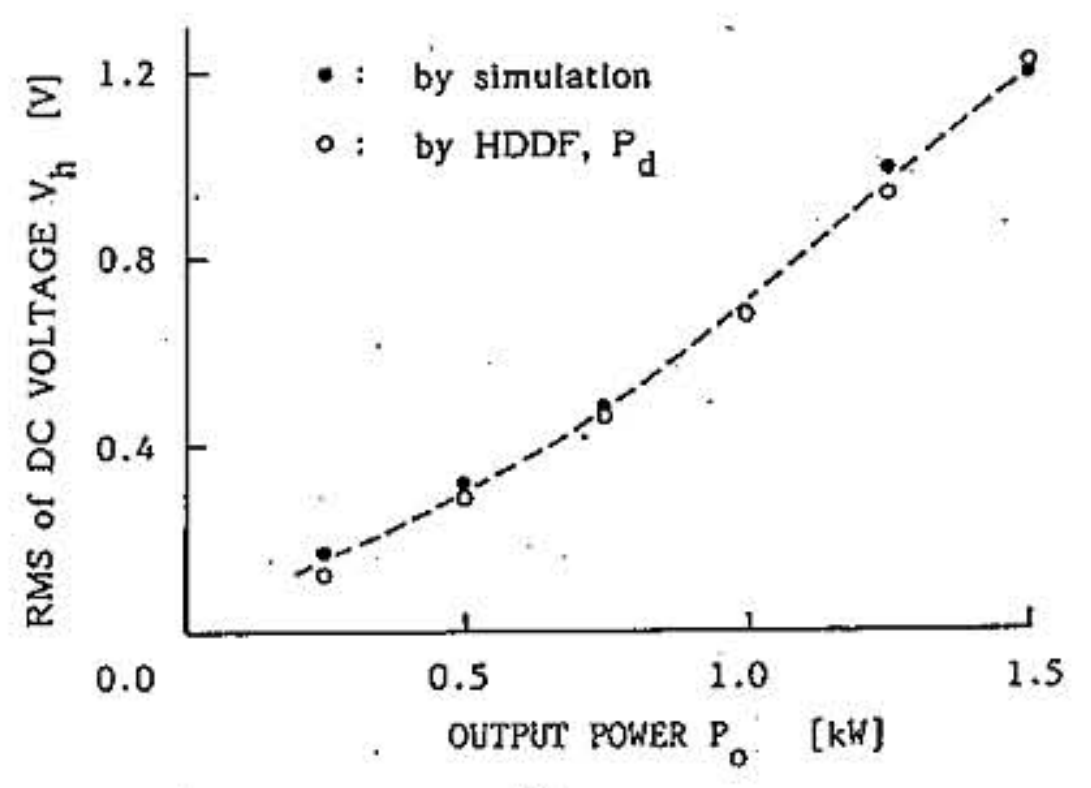

(b)

Fig. 5. Comparison of RMS values in rectifier operation obtained by simulation and by HDDF. (a) AC current harmonics, (b) DC voltage harmonics.

where $V_{\mathrm{dc} 0}$ and $I_{d 0}$ denote the average dc output voltage and the $d$-axis current, respectively; $L$ and $C$ denote the inductance of the ac reactor and capacitance of the dc capacitor, respectively. The RMS values of input harmonic current, $I_{h}$, and output harmonic voltage, $V_{h}$, obtained by simulation and by HDDF are compared in Fig. 5, where the original sinetriangular PWM method is used and the inverter operating conditions are $E=100[\mathrm{~V}], f=50[\mathrm{~Hz}], f_{s}=1.8[\mathrm{kHz}]$, $L=5[\mathrm{mH}], R=1[\Omega], C=330[\mu \mathrm{F}], V_{\mathrm{dc} 0}=160[\mathrm{~V}]$, $\omega_{s} / \omega=36$ and $L_{1}=1.6[\mathrm{mH}]$. To vary the output power, $R_{1}$ is changed while maintaining $\mathrm{PF}$ at unity and keeping $V_{\mathrm{dc}}$ constant. The modulation index $M$ and phase angle $\delta$ are simultaneously adjusted over the range, $M=0.862$ to 0.765 and $\delta=2.37$ to $19.5\left[^{\circ}\right]$, respectively and correspond to the power range $P_{0}=0.25$ to $1.5[\mathrm{~kW}] . I_{h}$ and $V_{h}$ are shown in terms of $P_{0}$ rather than in terms of $M$ because the range over which $M$ varies is too small. Both curves obtained by simulation and by HDDF are very close to each other.

Figs. 4 and 5 clearly show that once HDDF curves of the selected PWM method have been calculated, the harmonic characteristics of PWM inverter or PWM rectifier systems can be easily predicted without simulation.

\section{APPLICATION OF HDDF TO EVAluATE REAL TIME PWM METHODS}

\section{A. Analog PWM}

Here, HDDF curves of three typical real time analog PWM methods are compared and evaluated. They are the original sine-triangular PWM (SPWM) [1] shown in Fig. 6, har- 


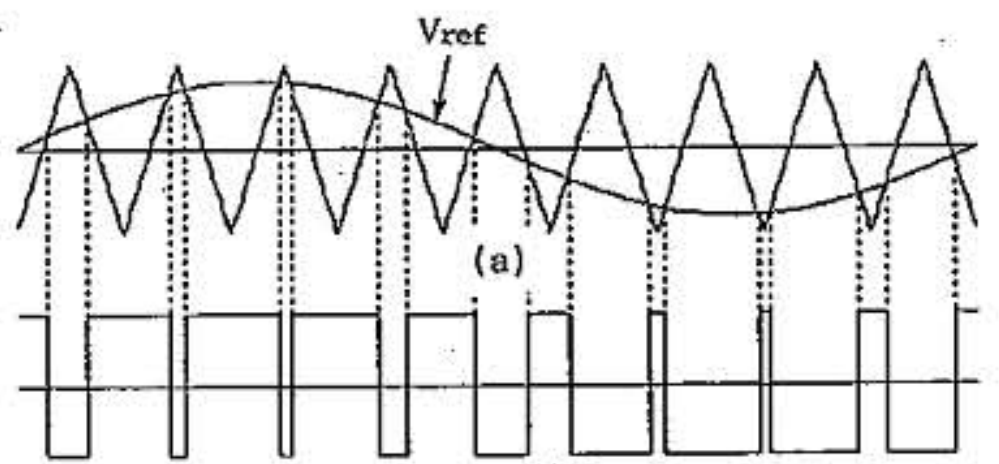

(b)

Fig. 6. Original sine PWM.

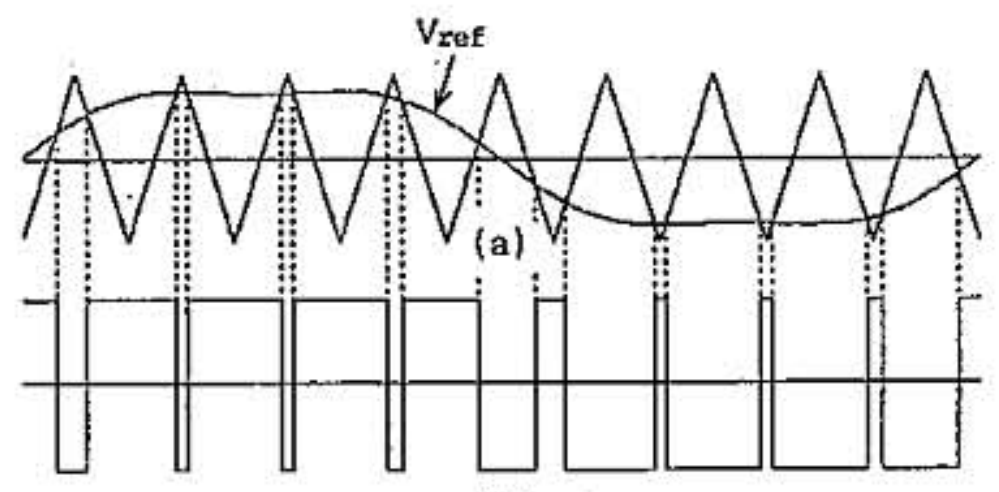

(b)

Fig. 7. Third-harmonic injected PWM.

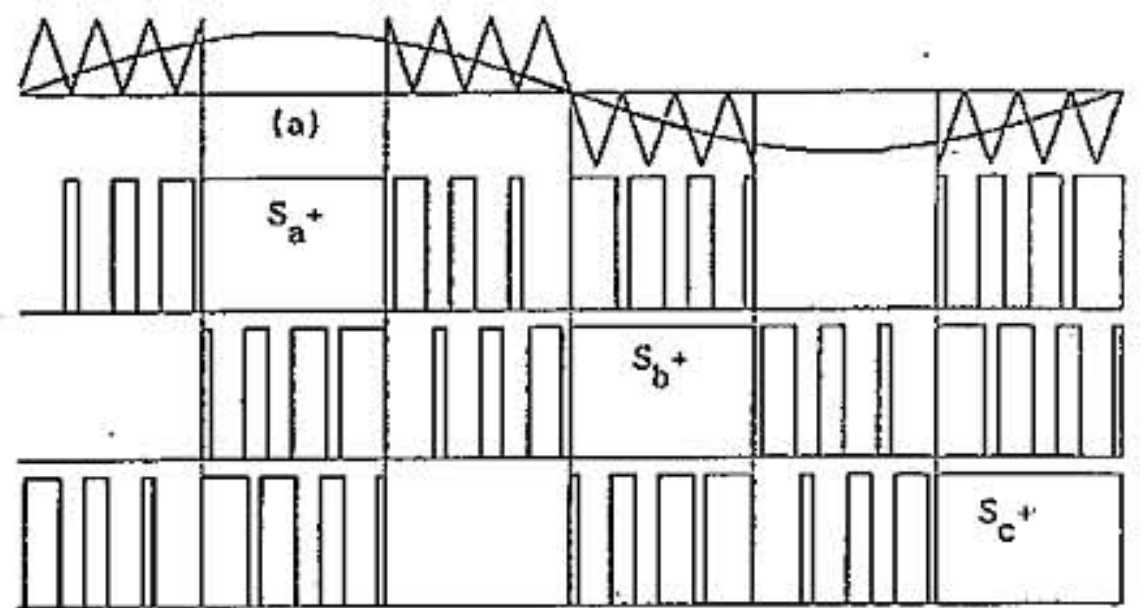

(b)

Fig. 8. Modified sine PWM. (a) MSPWM scheme. (b) Transistor gating signals.

monic injection PWM [2] (HIPWM), and modified sine PWM (MSPWM) [3]. HIPWM is derived from SPWM through the addition of the $17 \%$ third-harmonic component to the original sine reference waveform as shown in Fig. 7 , where $V_{\text {ref }}$ denotes the reference. The aim of injecting the third harmonic is to increase the maximum fundamental output voltage. Figs. 6 and 7, (b) shows the output potential for one phase. MSPWM defines the output on a line-to-line basis. Only the first and last $60^{\circ}$ intervals (per half-cycles) of the ac waveform are directly defined through intersections of respective sine and triangular waves as shown in Fig. 8, where (b) shows gating signals for each phase switch. The maximum output voltage by SPWM is $86.7 \%$ of that by HIPWM and MSPWM.

HDDF curves under variable voltage conditions are shown in Figs. 9 and 10, where the inverter output and carrier frequencies are $f=50[\mathrm{~Hz}]$ and $f_{s}=2.25[\mathrm{kHz}]$, respectively. The output voltage $V_{\text {nrm }}$ (normalized by $V_{\mathrm{dc}}$ ) for different PWM strategies and equivalent modulation index $M$ differ. Thus, for comparison purposes, it is more instructive to graph HDDF as a function of $V_{\mathrm{nrm}}$ rather than the modulation index $M$. From these figures, the following observations can be made:

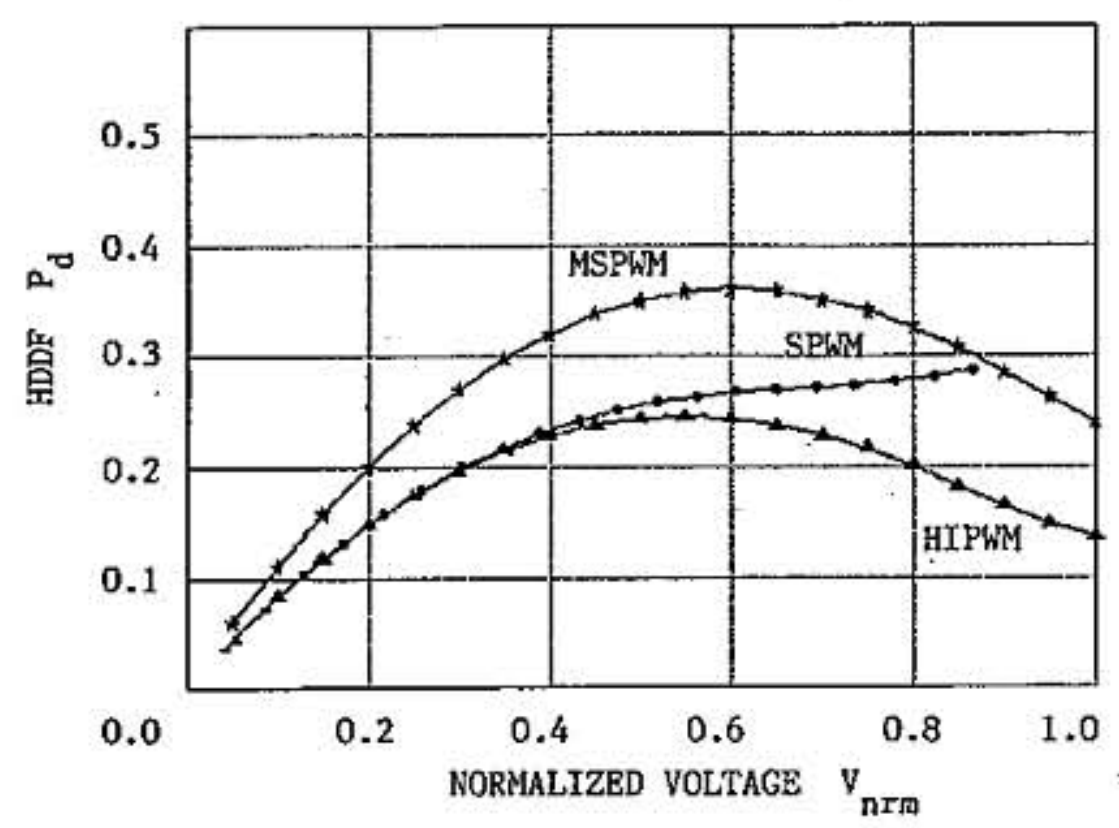

Fig. 9. Comparison of HDDF in the $d$-axis, $P_{d}$, for analog PWM's.

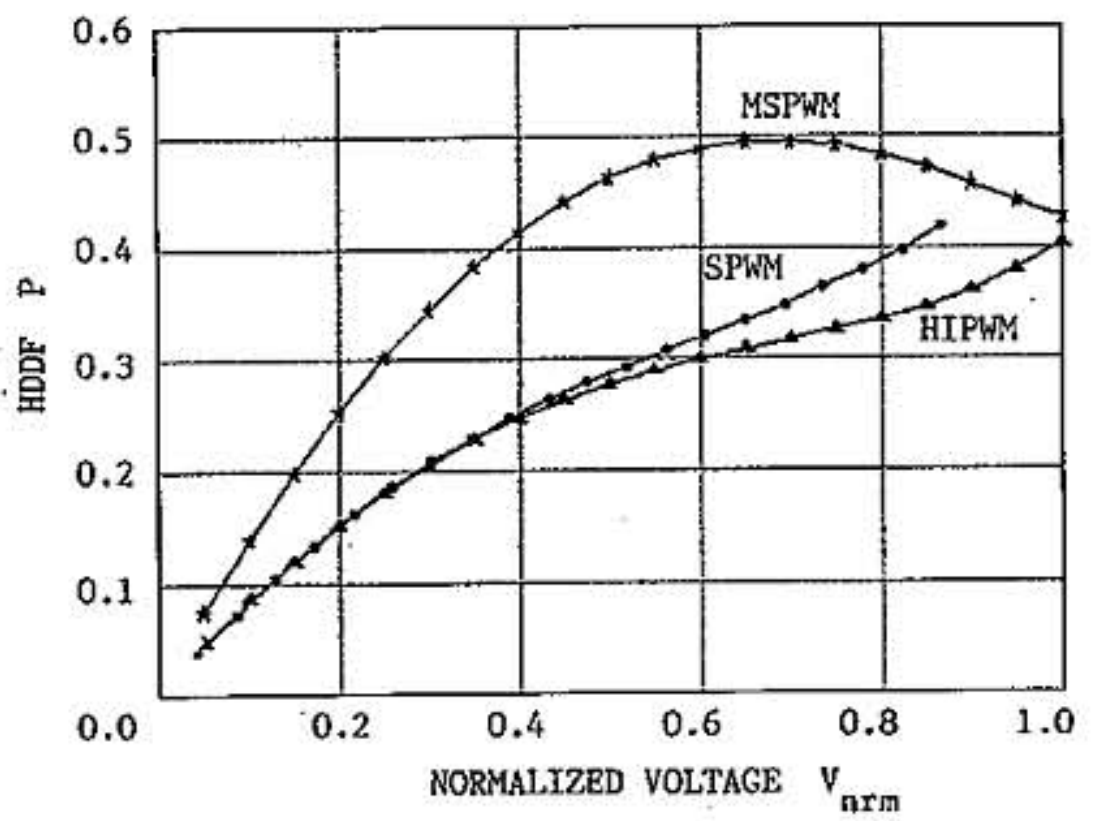

Fig. 10. Comparison of HDDF, $P$, for analog PWM's.

* SPWM and HIPWM show similar curves, but MSPWM is different. This stems from the different approach taken in creating the pulses.

* $P_{q}$ curves (not shown in the paper) of SPWM and HIPWM are almost identical.

* Of the two similar methods, HIPWM is superior to SPWM, especially in the high output voltage region.

* MSPWM has a higher maximum voltage than SPWM, but its characteristics are poorer except for the restricted high voltage region, $V_{\mathrm{nrm}}>0.8$.

In ac drives, suppression of torque ripples is important because of noise and vibration problems. From this point of view, HIPWM has an advantage over the others, especially in the high-speed region. HIPWM is also better in rectifier operation because it exhibits fewer dc voltage ripples than the others. As for the RMS of the harmonic current, there is not much difference between SPWM and HIPWM, but HIPWM is slightly better. On the whole, HIPWM is the best choice among the three.

\section{B. Digital PWM [4]}

Instantaneous space vector representation is employed to treat three-phase quantity as a whole. The instantaneous voltage vector is defined by 


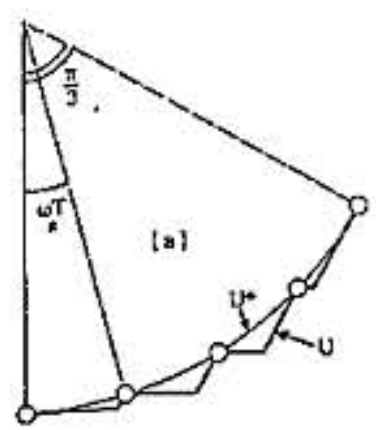

(a)

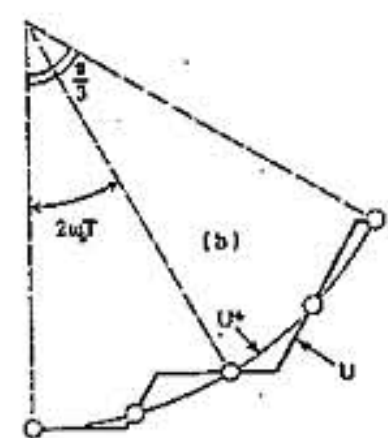

(b)

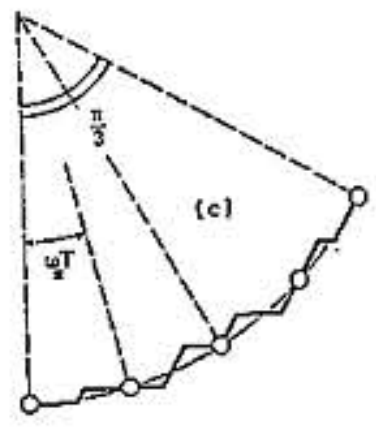

(c)
Fig. 11. Digital PWM's. (a) DPWM-A, (b) DPWM-C, and (c) DPWM-D.

$$
\begin{aligned}
\mathbf{V}_{n} & =\frac{V_{\mathrm{dc}}}{2}\left(S_{a}+\alpha S_{b}+\alpha^{2} S_{c}\right), & & \text { where } \alpha=\exp \left(j \frac{2 \pi}{3}\right) \\
& =V_{\mathrm{dc}} \exp \left\{j(n-1) \frac{2 \pi}{3}\right\} & & \text { for } n=1, \ldots, 6 \\
& =0 & & \text { for } n=0 \text { or } 7
\end{aligned}
$$

where $S_{\mathrm{a}}, S_{\mathrm{b}}$ and $S_{\mathrm{c}}$ are the switching functions for each phase. The inverter can produce eight voltage vectors, $V_{n}, n=$ $0,1, \ldots 7$, where $V_{0}$ and $V_{7}$ are so-called zero vectors. Consider the time-integral function of the inverter output vector as:

$$
\mathbf{U}=\int \mathbf{V}_{n} d t+\mathbf{U}_{0}
$$

The reference vector $U^{*}$, which is derived from the purely sinusoidal three phase output voltage, will be given by

$$
\mathbf{U}^{*}=-j \frac{\sqrt{3} V_{\mathrm{dc}} M}{2 \omega} \exp (j \omega t)
$$

where $M$ and $\omega$ denote the modulation index and output frequency of the inverter, respectively. The $U^{*}$ locus represents a perfect circle.

The PWM pattern is determined at every sampling interval so that $U$ may follow the $U^{*}$ locus as closely as possible. We call this means of creating pulses the quasi-circular-locus method (QCLM) and have proposed four PWM methods based on QCLM [7]. Here, HDDF curves of the four real time digital PWM's are compared and evaluated. They are the digital PWM of type-A (DPWM-A) to digital PWM of type-D (DPWM-D). With DPWM-A, vector $U$ moves along $U^{*}$ only from outside the reference circle as shown in Fig. 11(a), where "O" shows that a zero-vector is used. With DPWM-B, $U$ moves only from the inside the reference circle. With DPWMC, $U$ tracks $U^{*}$ from the inside at one sampling interval, and then from the outside at the next sampling interval, alternating as shown in Fig. 11(b). The switching functions for each phase and selected voltage vectors for two sampling intervals are shown in Fig. 12, where the pulse widths for the phase angle $0<\phi<\pi / 3$ are given by:

$$
\left.\begin{array}{rl}
\alpha_{1} & =M T \cos \left(\phi-\frac{\pi}{6}\right), \quad \alpha_{2}=M T \cos \left(\phi+\frac{\pi}{6}\right) \\
\beta & =\frac{T-\alpha_{1}}{2}
\end{array}\right\} .
$$

With DPWM-D, $U$ traces $U^{*}$ from the inside, and then from the outside alternately at every sampling interval as shown in Fig. 11(c).

HDDF curves under variable voltage conditions are shown in Figs. 13 and 14, where the inverter output and sampling

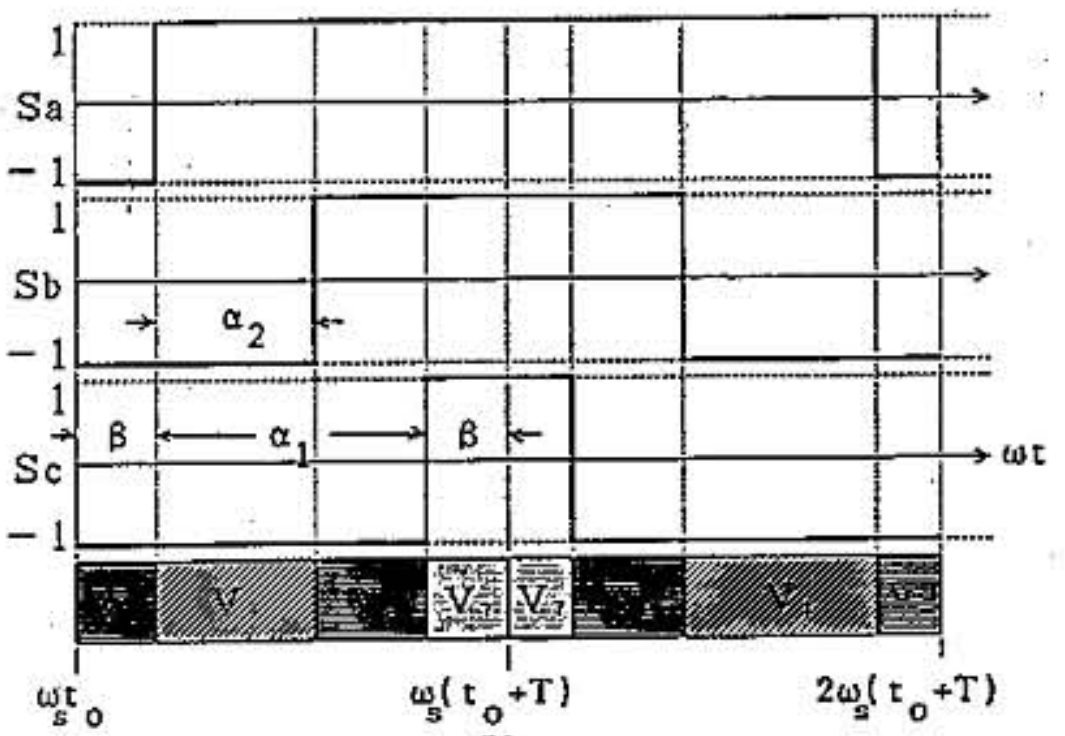

Fig. 12. Switching functions for each phase and voltage vectors used with DPWM-C for two sampling intervals.

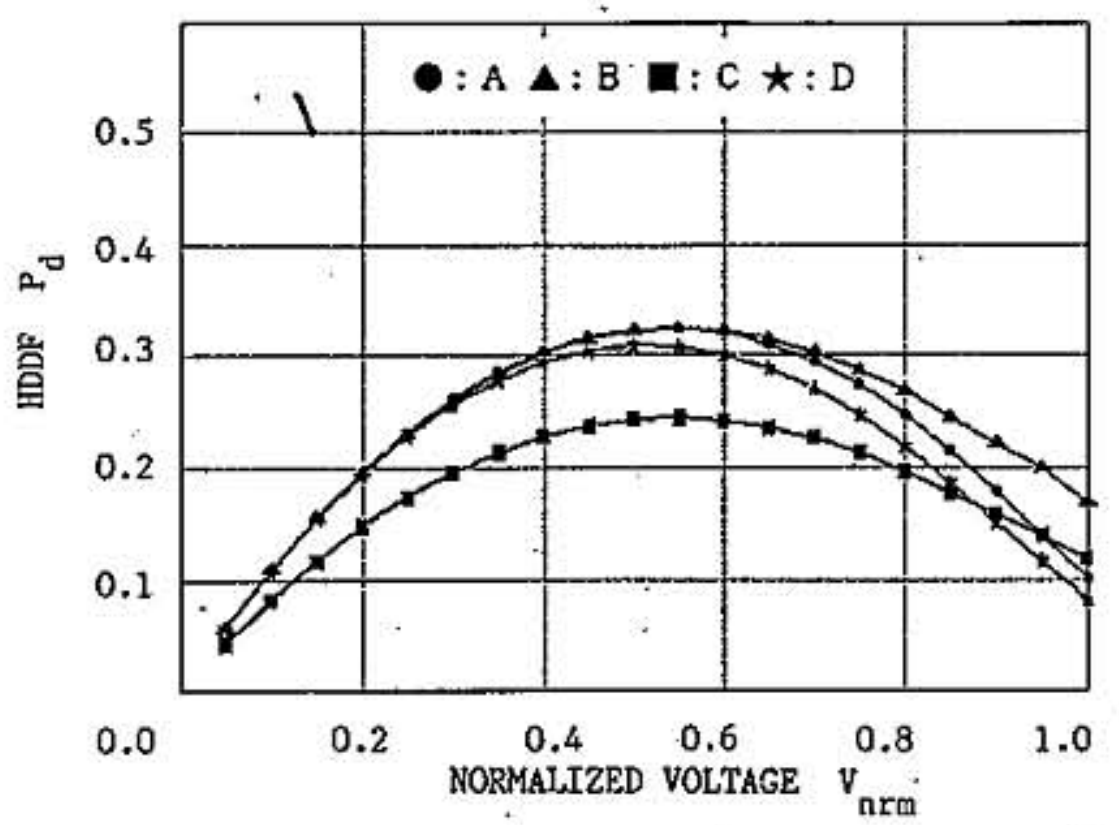

Fig. 13. Comparison of HDDF in the $d$-axis, $P_{d}$, for digital PWM's.

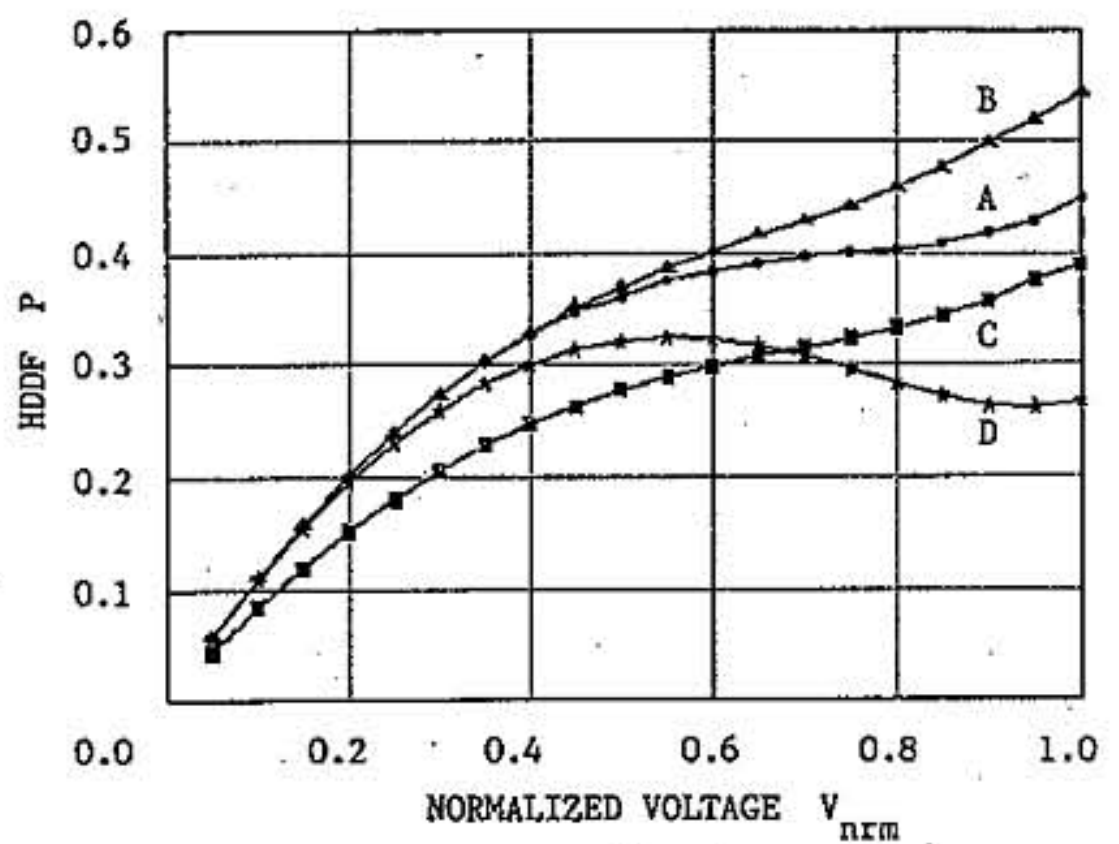

Fig. 14. Comparison of HDDF, $P$, for digital PWM's.

frequencies are $f=50[\mathrm{~Hz}]$ and $f_{s}=1: 8[\mathrm{kHz}]$, respectively. From these figures, the following can be observed:

* DPWM-A and -B provide similar HDDF curves, but DPWM-A is slightly better.

* DPWM-C and D are superior to DPWM-A and B.

* DPWM-D has an advantage over DPWM-C only in the high voltage region, $V_{\mathrm{nrm}}>0.7$ for $P$ or $V_{\mathrm{nrm}}>0.9$ for $P_{d}$.

* If considered on the whole, DPWM-C has the best characteristics of the four.

Therefore, for ac drives, DPWM-C is superior to the others because it produces the fewest torque ripples among the four 
methods. As for the RMS of the harmonic current, DPWM-C is best in the voltage range $V_{\text {nrm }}<0.7$ but DPWM-D is the best in the range $V_{\mathrm{nrm}}>0.7$. DPWM-A and B are poor.

If analog PWM's and digital PWM's are compared, the following can be observed:

* Digital PWM's provide lower $P_{d}$ curves than analog PWM's in the high voltage region. Therefore, digital PWM's have an advantage for AC drive applications over analog ones in the same region.

* DPWM-C and HIPWM have almost identical HDDF curves. Thus DPWM-C is a digital alternative of HIPWM.

* If an analog PWM is adopted, HIPWM would be the best choice, and if a digital PWM is adopted, method C would be the best choice.

\section{Normalization of Switching Frequency}

As for the analog PWM's, each phase has one switchtransition at every sampling time because each phase is controlled independently. As for the digital PWM's, however, the average switching number depends on the individual method because the three phases are not treated independently. Therefore, normalization of switching frequency is necessary to evaluate PWM methods. The average switching frequency, $f_{\text {sw }}$ of each method is:

$$
\begin{array}{ll}
f_{\mathrm{sw}}=f_{\mathrm{c}} & \text { for the analog PWM's, } \\
f_{\mathrm{sw}}=(2 / 3) f_{s} & \text { for DPWM-A, DPWM-B and DPWM-D, and } \\
f_{\mathrm{sw}}=(1 / 2) f_{s} & \text { for DPWM-C. }
\end{array}
$$

where $f_{c}$ and $f_{s}$ are the carrier and sampling frequencies, respectively. The HDDF curves in Figs. 13 and 14 are obtained by multiplying the original HDDF values by the coefficients $1 / 2$ for DPWM-C and $2 / 3$ for DPWM-A, B, and D, taking SPWM as the basis.

\section{CONCLUSION}

The harmonic distortion determining factor (HDDF), which represents the intrinsic spectral properties of PWM methods, is introduced. HDDF is almost independent of the inverter operating conditions such as the switching frequencies or load parameters if the switching frequency is 40 times greater than the output frequency. Furthermore, it has a close relation to the RMS values of the harmonic current and motor torque ripples for induction motor drives, or RMS values of the harmonic current and dc voltage ripples for rectifier operation. Therefore, it is useful as a common quality index for the evaluation of PWM methods.

Three typical analog PWM methods and four digital PWM methods are compared and evaluated based on HDDF values. It is shown that the harmonic injected PWM for analog PWM, or the digital PWM of type $\mathrm{C}$ have the best characteristics.

\section{ACKNOWLEDGMENT}

The authors wish to express their sincere appreciation to S. Sano, the former student, for his eager cooperation in completing this paper.

\section{REFERENCES ,}

[1] R. Bonnert and R. Wu, "Improved three phase pulse width modulation for over modulation," in Conf. Rec. 1984 IEEE Ind. Applicat. Soc. Ann. Meeting.

[2] J. Houldsworth and D. Grant, "The use of harmonic distortion to increase the output of a three-phase PWM inverter," IEEE Trans. Ind. Applicat., vol. 20 , no. 5, pp. 1224-1228, Sept./Oct. 1984.

[3] T. Ohnishi and H. Okitsu, "A novel PWM technique for three phase inverter/converter," in Conf. Rec. of 1983 IPEC-Tokyo, pp. 384-395.

[4] S. Fukuda, Y. Iwaji, and H. Hasegawa, "PWM technique for inverter with sinusoidal output current," IEEE Trans. Power Electron., vol. 5, no. 1, pp. 54-61, Jan. 1990.

[5] H. W. Van der Broeck, H.-Ch. Skudelny, and G. V. Stanke, "Analysis and realization of a pulsewidth modulator based on voltage space vectors," IEEE Trans. Ind. Applicat., vol. 24 , no. 1, pp. 142-150, Jan./Feb. 1988.

[6] Y. Iwaji and S. Fukuda, "PWM waveform analysis using harmonic distortion determining factor for induction motor drives," in Conf. Rec. 1991 IEEJ Hokkaido Branch Conv., pp. 72-73.

[7] _ "Waveform analysis of PWM inverters using the harmonic distortion determining factor," in Conf. Rec. 1993 IEEJ National Conv., pp. $110-111$.

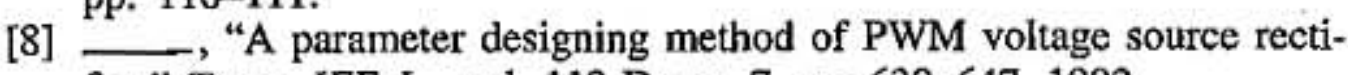
fier," Trans. IEE J. , vol. 112-D, no. 7, pp. 639-647, 1992.

[9] _ "PWM waveform analysis using harmonic distortion determining factor for voltage source rectifiers," in Conf. Rec. 1991 IEEJ Hokkaido Branch Conv., pp. 74-75.

[10] M. Boost and P. Ziogas, "State-of-the-art carrier PWM technique: A critical evaluation," IEEE Trans. Ind. Applicat., vol. 24, no. 2, pp. 271-280, Mar./Apr. 1988.

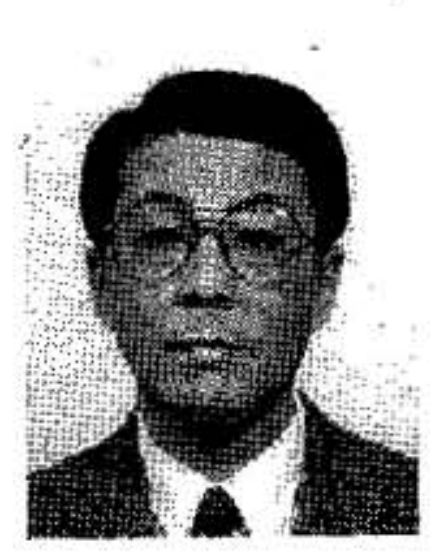

Shoji Fukuda (M'85) received the B.E.E., M.S.F.E., and Ph.D. degrees from Hokkaido University, Sapporo, Japan, in 1965, 1967, and 1977 , respectively.

In 1967, he joined the Faculty of Engineering, Hokkaido University, where he is currently an Associate Professor in the Department of Electrical Engineering. From 1981-1983, he worked at the University of Saskatchewan, Canada, as a postdoctoral fellow. He has been engaged in the research of microprocessor-based PWM control of rectifiers/inverters, ac drives, and active power filters.

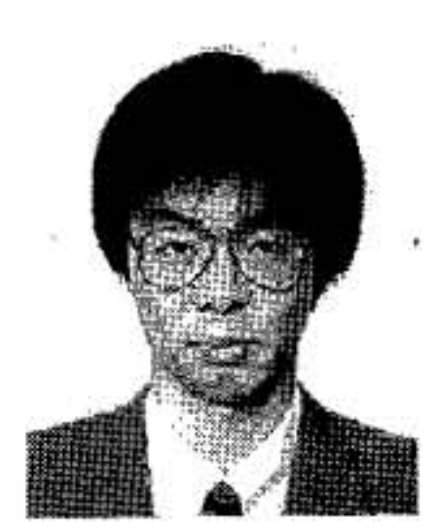

Yoshitaka Iwaji received the B.E.E. degree from Ibaraki University, Hitachi, Japan, in 1987, and the M.S.E.E. and Ph.D. degrees from Hokkaido University, Sapporo, Japan, in 1989 and 1992, respectively.

In 1992, he joined Hitachi Research Laboratory, Hitachi Ltd., Ibaraki, Japan, and has been engaged in research on PWM inverters for industrial applications. 